\title{
Streptococcal Toxic Shock Syndrome Caused by Group G Streptococcus, United Kingdom
}

\section{Melissa Baxter, Marina Morgan}

We describe successful management of 3 patients with streptococcal toxic shock syndrome (STSS) attributable to group G Streptococcus infection. This small series supports recognition of group G Streptococcus in the etiology of STSS. We propose intravenous immunoglobulin be used in treatment as it is for STSS caused by group A Streptococcus.

\begin{abstract}
Ctreptococcal toxic shock syndrome (STSS) was defined in 1993 as the isolation of group A Streptococcus (GAS) with parameters indicative of multiorgan dysfunction (1). STSS remains associated with high mortality rates, even with adequate antimicrobial treatment (2). Recently, 12 invasive infections with $\beta$-hemolytic streptococci of Lancefield group $\mathrm{G}$ also fulfilling the criteria of STSS have been reported (mortality rate $83 \%$ ) (3-12). The benefit of adjunctive intravenous immunoglobulin (IVIg) in the treatment of STSS is difficult to prove because the limited number of cases precludes results of sufficient statistical power. Observational studies $(2,14)$ and 1 small, early terminated, randomized control trial (15) have demonstrated improved survival among patients with GAS STSS treated with IVIg. We describe 3 patients treated in an 880-bed teaching hospital during 2009-2014; all 3 patients had illness meeting the case definition of STSS that were caused by invasive group G Streptococcus (GGS) infection. Successful treatment included IVIg, providing further support for the use of IVIg in treating STSS.
\end{abstract}

\section{The Patients}

In 2009, a 46-year-old woman (patient 1) with multiple sclerosis and lower limb lymphedema was admitted to the hospital with a 1-week history of fever, vomiting, rigors, sore throat, and erythema of the right leg with a healing cut on the foot. Patchy cellulitis extended from the foot to groin and was associated with elevated body temperature $\left(39.4^{\circ} \mathrm{C}\right)$. Her creatine kinase level was high at 3,951 IU/L, and she had an elevated C-reactive protein (CRP) level of $396 \mathrm{mg} / \mathrm{L}$. Her illness was initially managed as severe cellulitis. She received intravenous fluids, clindamycin $(1.2 \mathrm{~g}$ every $6 \mathrm{~h})$, and flucloxacillin $(1 \mathrm{~g}$ every $6 \mathrm{~h})$. However, her condition rapidly deteriorated. She had onset of

Author affiliation: Royal Devon and Exeter National Health Service Foundation Trust, Devon, United Kingdom

DOI: http://dx.doi.org/10.3201/eid2301.161009 hypotension (blood pressure $77 / 24 \mathrm{~mm} \mathrm{Hg}$ ) refractory to $8 \mathrm{~L}$ fluid resuscitation, prompting transfer to the intensive therapy unit (ITU) for cardiovascular support. Computed tomography scan results were compatible with adult respiratory distress syndrome. Shock and spreading cellulitis (with patches appearing on the contralateral leg) suggested streptococcal myositis or multifocal necrotizing fasciitis. Flucloxacillin was changed to intravenous daptomycin (4 $\mathrm{mg} / \mathrm{kg}$ daily), and meropenem ( $1 \mathrm{~g}$ every $8 \mathrm{~h}$ ) and $2 \mathrm{~g} /$ $\mathrm{kg}$ polyclonal intravenous immunoglobulin (IVIg) (Privigen; CSL Behring, West Sussex, UK) were administered. Her condition continued to deteriorate. Erythema spread beyond skin markings, her CRP plateaued at levels $>300$ $\mathrm{mg} / \mathrm{L}$, and her serum IgG level was $4.6 \mathrm{mg} / \mathrm{L}$, prompting administration of a second dose of IVIg $2 \mathrm{~g} / \mathrm{kg}$ on day 3 . Surgical exploration released $30 \mathrm{~mL}$ of fluid and revealed macroscopically normal fascia and muscle. Histologic evaluation revealed severely necrotic soft tissue. GGS was isolated from all operative specimens. A transthoracic echocardiogram showed no vegetations. Three weeks after admission, the patient was discharged on prophylactic penicillin and remains well.

In 2011, a 63-year-old previously healthy man (patient 2) was admitted to the hospital with a 5-day history of confusion, headache, and bilateral swollen painful knees. Among purpuric lesions on the left lower leg was a small healing wound. Multiorgan failure and septic shock necessitated transfer to the ITU for inotropic support and hemofiltration. Blood tests revealed a reduced platelet count of $54 \times 10^{9} / \mathrm{L}$ and elevated levels of creatinine at $210 \mu \mathrm{mol} / \mathrm{L}$, serum lactate at $10.2 \mathrm{mmol} / \mathrm{L}, \mathrm{CRP}$ at $353 \mathrm{mg} / \mathrm{L}$, and creatine kinase at 2,080 IU/L. Culture of joint fluid from each knee revealed GGS. The patient was treated empirically with intravenous imipenem (500 mg every $12 \mathrm{~h}$ ), clindamycin $(1.8 \mathrm{~g}$ every $6 \mathrm{~h})$, vancomycin ( $1 \mathrm{~g}$ stat $)$, and $2 \mathrm{~g} / \mathrm{kg}$ polyclonal IVIg (Privigen). Despite dramatic clinical improvement, a static CRP level and worsening pain in other joints necessitated repeated arthroscopic washouts of his knees, wrists, hips, and 1 shoulder. Although gram-positive cocci were observed on microscopic evaluation of the knee and shoulder fluids, all aspirates and blood cultures were sterile. A transesophageal echocardiogram found no evidence of endocarditis; however, a repeat transesophageal echocardiogram 1 month after admission revealed a vegetation, necessitating aortic valve replacement. The patient was discharged after 13 weeks and remains well. 
In 2014, a 66-year-old man (patient 3) with a history of diabetes mellitus was admitted to the hospital in septic shock. A total knee replacement 6 months earlier had resulted in chronic leg lymphedema. Noteworthy blood test results included a high serum lactate level of $4 \mathrm{mmol} / \mathrm{L}$, an elevated creatinine level of $318 \mu \mathrm{mol} / \mathrm{L}$, and a high alanine aminotransferase level of $210 \mathrm{IU} / \mathrm{L}$. The patient had a blood pressure of $40 / 20 \mathrm{~mm} \mathrm{Hg}$, rapidly spreading erythema from the knee to the abdomen, and suffused conjunctivae. His illness was managed clinically as toxic shock syndrome. He received inotropic support, mechanical ventilation, hemofiltration, and intravenous linezolid $(600 \mathrm{mg}$ every 12 h), intravenous clindamycin (1.2 g every $6 \mathrm{~h})$, and $2 \mathrm{~g} / \mathrm{kg}$ polyclonal IVIg (Privigen). Arthroscopic washout of the prosthetic knee revealed copious pus that yielded GGS on culture. Although cardiovascular function stabilized, blood parameters continued to deteriorate. On day 3, following open debridement of the knee, the patient began to improve, and his CRP level remained elevated at $280 \mathrm{mg} / \mathrm{L}$. Antimicrobial drugs were changed to intravenous daptomycin (7 $\mathrm{mg} / \mathrm{kg}$ once daily) to maximize antibiofilm activity (reference 16 in online Technical Appendix, http://wwwnc.cdc. gov/EID/article/23/1/16-1009-Techapp1.pdf). After a negative echocardiography, the patient was discharged home on day 30 , completing a 6-week course of daptomycin as an outpatient, and successfully retained the knee prosthesis. He remains well on life-long penicillin prophylaxis.

\section{Conclusions}

We describe 3 patients with group G STSS. Isolates were identified by laboratory serotyping of the group $\mathrm{G}$ carbohydrate surface antigen. All 3 patients had a favorable outcome after aggressive therapy with a combination of antiexotoxin antibiotics, IVIg, and surgical intervention.

The emergence of GGS causing skin and soft tissue infections (reference 17 in online Technical Appendix) might reflect improved detection, increased virulence, or a growing population of immunocompromised hosts. Similar to GAS, GGS shares multiple virulence factors, including streptokinase, fibronectin, IgG binding proteins, streptolysin $\mathrm{O}, \mathrm{C} 5 \mathrm{a}$ peptidase (reference 18 in online Technical Appendix), and antiphagocytic M proteins, of which emm types stg10 and $\operatorname{stg} 2078$ of GGS are significantly associated with invasive disease (reference 19 in online Technical Appendix). Given clinical presentations of STSS by group G and group C Streptococcus are indistinguishable from group A STSS, the underlying mechanisms are probably related. However, unlike group A STSS, with group G STSS, underlying co-morbidities predominate, including cardiopulmonary disease, diabetes mellitus, malignancy, or hepatic failure (6).

IVIg has superantigen neutralizing activity (reference 20 in online Technical Appendix) and reduces mortality rates in group A STSS $(2,14,15)$ A recent review of IVIg use for severe sepsis and septic shock yielded little evidence of benefit (reference 21 in online Technical Appendix). However, in that review, there was no discrimination between superantigen, exotoxin-driven, gram-positive sepsis and primarily endotoxin-driven, gram-negative sepsis, and very few cases of gram-positive sepsis were included (reference 21 in online Technical Appendix).

Twelve cases of group G STSS have been previously documented (3-12); 10 of those patients died (mortality rate $83 \%$ ). With the addition of the 3 cases we reported, group G STSS has an overall reported mortality rate of $66 \%$. The use of IVIg was described in the cases of 4 patients, 3 of whom died $(5,7,11,12)$, including 1 who received low-dose IVIg (400 mg/kg) (12); for the other patients, the IVIg dosage was unspecified. Although our case series is small, it describes long-term survival in 3 patients with group $G$ STSS. We propose that the definition of STSS extends beyond that of a condition exclusive to GAS infection and recommend that polyclonal immunoglobulin be considered as a potentially lifesaving adjunctive therapy.

Dr. Baxter and Dr. Morgan are clinical microbiologists working at the Royal Devon and Exeter National Health Service Foundation Trust. Both have a particular interest in the diagnosis and management of necrotizing skin, soft tissue infections, and toxic shock.

\section{References}

1. Breiman RF. Defining the group A streptococcal toxic shock syndrome. Rationale and consensus definition. JAMA. 1993; 269:390-1. http://dx.doi.org/10.1001/jama.1993.03500030088038

2. Linnér A, Darenberg J, Sjölin J, Henriques-Normark B, Norrby-Teglund A. Clinical efficacy of polyspecific intravenous immunoglobulin therapy in patients with streptococcal toxic shock syndrome: a comparative observational study. Clin Infect Dis. 2014;59:851-7. http://dx.doi.org/10.1093/cid/ciu449

3. Kugi M, Tojo H, Haraga I, Takata T, Handa K, Tanaka K. Toxic shock-like syndrome caused by group G Streptococcus. J Infect. 1998;37:308-9. http://dx.doi.org/10.1016/S0163-4453(98)92510-5

4. Shimomura Y, Okumura K, Murayama SY, Yagi J, Ubukata K, Kirikae T, et al. Complete genome sequencing and analysis of a Lancefield group G Streptococcus dysgalactiae subsp. equisimilis strain causing streptococcal toxic shock syndrome (STSS). BMC Genomics. 2011;12:17. http://dx.doi.org/10.1186/1471-2164-12-17

5. Barnham MRD, Weightman NC, Anderson AW, Tanna A. Streptococcal toxic shock syndrome: a description of 14 cases from North Yorkshire, UK. Clin Microbiol Infect. 2002;8:174-81. http://dx.doi.org/10.1046/j.1469-0691.2002.00396.x

6. Hashikawa S, Iinuma Y, Furushita M, Ohkura T, Nada T, Torii K, et al. Characterization of group C and G streptococcal strains that cause streptococcal toxic shock syndrome. J Clin Microbiol. 2004;42:186-92. http://dx.doi.org/10.1128/ JCM.42.1.186-192.2004

7. Nei T, Akutsu K, Shima A, Tsuboi I, Suzuki H, Yamamoto T, et al. A case of streptococcal toxic shock syndrome due to Group G streptococci identified as Streptococcus dysgalactiae subsp. equisimilis. J Infect Chemother. 2012;18:919-24. http://dx.doi.org/10.1007/s10156-012-0375-x 
8. Hagiya H, Okita S, Kuroe Y, Nojima H, Otani S, Sugiyama J, et al. A fatal case of streptococcal toxic shock syndrome due to Streptococcus dysgalactiae subsp. equisimilis possibly caused by an intramuscular injection. Intern Med. 2013;52:397-402. http://dx.doi.org/10.2169/internalmedicine.52.8846

9. Horii T, Izumida S, Takeuchi K, Tada T, Ishikawa J, Tsuboi K. Acute peritonitis and salpingitis associated with streptococcal toxic shock syndrome caused by Lancefield group $\mathrm{G}$ alphahaemolytic Streptococcus dysgalactiae subsp. equisimilis. J Med Microbiol. 2006;55:953-6. http://dx.doi.org/10.1099/ jmm.0.46507-0

10. Takagi K, Shimizu H, Iwasaki H, Tsutani H, Ueda T. Serum cytokine level during continuous venovenous hemofiltration in toxic shock-like syndrome due to group $\mathrm{G}$ beta Streptococcus bacteremia in a patient with idiopathic thrombocytopenic purpura. Scand J Infect Dis. 2002;34:403-6. http://dx.doi.org/10.1080/00365540110080683

11. Hirose Y, Yagi K, Honda H, Shibuya H, Okazaki E. Toxic shock-like syndrome caused by non-group A beta-hemolytic streptococci. Arch Intern Med. 1997;157:1891-4. http://dx.doi.org/10.1001/archinte.1997.00440370141015

12. Wagner JG, Schlievert PM, Assimacopoulos AP, Stoehr JA, Carson PJ, Komadina K. Acute group G streptococcal myositis associated with streptococcal toxic shock syndrome: case report and review. Clin Infect Dis. 1996;23:1159-61. http://dx.doi.org/10.1093/clinids/23.5.1159

13. Mylvaganam H, Bruun T, Vindenes HA, Langeland N, Skrede S. Molecular epidemiological investigation of an outbreak of invasive beta-haemolytic streptococcal infection in western Norway. Clin Microbiol Infect. 2009;15:245-52. http://dx.doi.org/10.1111/j.1469-0691.2008.02664.x

14. Kaul R, McGeer A, Norrby-Teglund A, Kotb M, Schwartz B, O'Rourke K, et al. Intravenous immunoglobulin therapy for streptococcal toxic shock syndrome - a comparative observational study. Clin Infect Dis. 1999;28:800-7. http://dx.doi.org/10.1086/515199

15. Darenberg J, Ihendyane N, Sjölin J, Aufwerber E, Haidl S, Follin P, et al. Intravenous immunoglobulin $\mathrm{G}$ therapy in streptococcal toxic shock syndrome: a European randomized, double-blind, placebo-controlled trial. Clin Infect Dis. 2003;37:333-40. http://dx.doi.org/10.1086/376630

Address for correspondence: Melissa Baxter, Department of Microbiology, Royal Devon and Exeter NHS Foundation Trust, Exeter, Devon EX3 5DW, UK; email: melissabaxter1@nhs.net

\section{February 2015: Complicated Datasets}

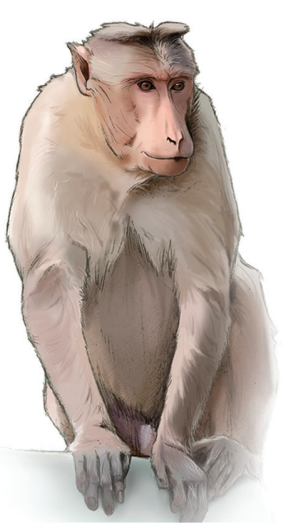

- Entry Screening for Infectious Diseases in Humans

- Timing of Influenza A(H5N1) in Poultry and Humans and Seasonal Influenza Activity Worldwide, 2004-2013

- Quantifying Reporting Timeliness to Improve Outbreak Control

- Tickborne Relapsing Fever, Bitterroot Valley, Montana, USA

- Simulation Study of the Effect of Influenza and Influenza Vaccination on Risk of
Acquiring Guillain-Barré Syndrome

- Evidence for Elizabethkingia anophelis Transmission from Mother to Infant, Hong Kong

- Microbiota that Affect Risk for Shigellosis in Children in Low-Income Countries

- Optimizing Distribution of Pandemic Influenza Antiviral Drugs

- pH Level as a Marker for Predicting Death among Patients with Vibrio vulnificus Infection, South Korea, 2000-2011

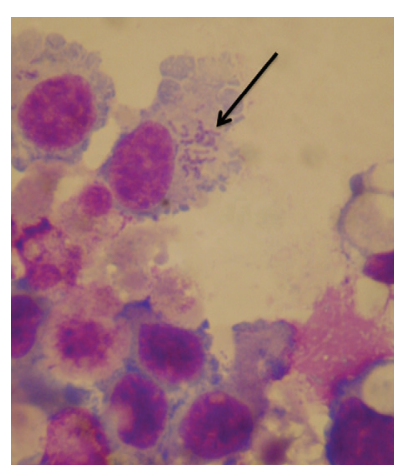

- Vesicular Stomatitis VirusBased Vaccines against Lassa and Ebola Viruses

- Use of Insecticide-Treated House Screens to Reduce Infestations of Dengue Virus Vectors, Mexico

- Comparative Analysis of African Swine Fever Virus Genotypes and Serogroups

- Refining Historical Limits Method to Improve Disease Cluster Detection, New York City, New York, USA

- Naturally Acquired Antibodies against Haemophilus influenzae Type a in Aboriginal Adults, Canada

- Infectious Causes of Encephalitis and Meningoencephalitis in Thailand, 2003-2005

- Lagenidium giganteum Pathogenicity in Mammals

- Novel Reassortant Influenza A(H5N8) Viruses among Inoculated Domestic and Wild Ducks, South Korea, 2014
- Murine Typhus, Reunion, France, 2011-2013

- Awareness and Support of Release of Genetically Modified "Sterile" Mosquitoes, Key West, Florida, USA

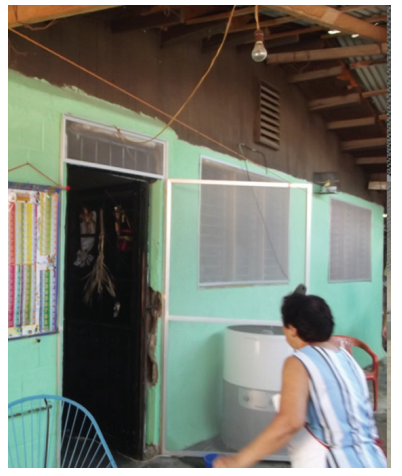

